\title{
Effects of bufotenine and p-chlorophenylalanine on reactivity to footshock*
}

\author{
GORDON WINOCUR \\ Trent University, Peterborough, Ontario, Canada \\ and \\ S. P. BAGCHI \\ Department of Psychiatric Research, University Hospital, Saskatoon, Saskatchewan, Canada
}

\begin{abstract}
In two experiments, groups of rats were injected with bufotenine, p-chlorophenylalanine (PCPA), bufotenine + PCPA (B + PCPA), or saline, and their reactions to footshock were recorded during tests of distractability and response thresholds. Bufotenine consistently decreased sensitivity to shock, while PCPA produced the opposite effect. Individual effects of bufotenine and PCPA were not apparent in the B + PCPA groups, which, in both experiments, behaved like saline control groups. The results support the hypothesis that bufotenine and PCPA affect reactivity to external stimuli in opposite ways.
\end{abstract}

Although the importance of serotonin for normal brain function has been recognized for some time, less attention has focused on the various derivatives of serotonin. Of particular interest are the $\mathrm{N}, \mathrm{N}$-dimethyl compounds, which, in addition to being structurally similar to serotonin, are all psychomimetic. Of this group, bufotenine (5-hydroxy-N, N-dimethyltryptamine) most closely resembles serotonin and is the most psychoactive.

The hallucinogenic effects of bufotenine, although less potent, have been compared with those of lysergic acid diethylamide (LSD) (Evarts, 1956; Fabing \& Hawkins, 1956). Bufotenine and LSD are also related on the basis of cross-tolerance, which suggests that their respective effects are mediated by a common mechanism, probably involving serotonin. The clinical importance of bufotenine has also been emphasized in reports demonstrating the presence of bufotenine in unusually high proportions in the urine of psychotic patients (Himwich \& Brune, 1966).

Behavioral research involving bufotenine has been more limited, but the available evidence indicates that intraperitoneal injections of the drug tend to interfere with the normal function of sensory and perceptual processes. For example, Maire and Hixon (1966) found bufotenine-injected rats to be impaired on various learning tasks and attributed the deficit to an ineffective use of available stimulus cues. Investigations in our laboratory have similarly demonstrated that rats under the influence of bufotenine do not respond normally to environmental stimuli, particularly under conditions of stress (Winocur, Bagchi, \& Young, 1969; Winocur, Bagchi, \& Hubbard, 1971). For example, bufotenine-injected rats were found to be unresponsive to frustrative events which, in normal animals, produced

*Requests for reprints should be sent to Gordon Winocur, Trent University, Peterborough, Ontario, Canada. intensified goal-directed behavior.

The present research continues the investigation into the relationship between bufotenine and sensory functions by examining the effects of bufotenine on reactions to aversive stimulation. In Experiment $I$, the distractability of drug and control groups of rats was compared by introducing a novel aversive stimulus during the performance of a well-established and consistently rewarded response. A second objective was to examine further an antagonistic relationship between bufotenine and p-chlorophenylalanine (PCPA), a depletor of brain serotonin (Koe \& Weissman, 1966). Several reports have shown that PCPA by itself has an excitatory effect on various types of behavior (Tenen, 1967; Stevens, 1970; Brody, 1970). Winocur et al (1971) reported that the individual effects of PCPA and bufotenine were eliminated when the two drugs were administered in combination. Accordingly, in the subsequent experiments, the performance of bufotenine-injected groups of rats is compared with that of PCPA and bufotenine + PCPA (B + PCPA) groups.

\section{EXPERIMENT I}

\section{Method}

Subjects. Male albino Sprague-Dawley rats, weighing approximately $250 \mathrm{~g}$, were used as Ss. The animals were housed individually in wire mesh cages with food available at all times.

The Ss were divided into bufotenine (B) $(\mathrm{N}=8)$, PCPA $(\mathrm{N}=$ $8)$, and B + PCPA $(\mathrm{N}=8)$ groups. Twelve animals were assigned in equal numbers to three saline control groups $(a, b, c)$ to control for the effects of varying numbers of injections.

Apparatus. The apparatus was a wooden chamber $(10 \mathrm{ft}$ in length), consisting of a startbox (6 $\times 4 \times 5 \mathrm{in}$.), a runway ( $9 \mathrm{ft} \times$ 4 in. $\times 5$ in.), and a goalbox ( $6 \times 4 \times 5$ in.). A guillotine door separated the startbox from the runway. The inside of the apparatus was painted flat black and was covered with a hinged Plexiglas ceiling. The floor of the apparatus consisted of wood, painted flat black except for a 1-ft length of grid floor situated about half way down the alley. The grids were attached to a BRS constant current shock generator scrambler (SGS-003). A series 


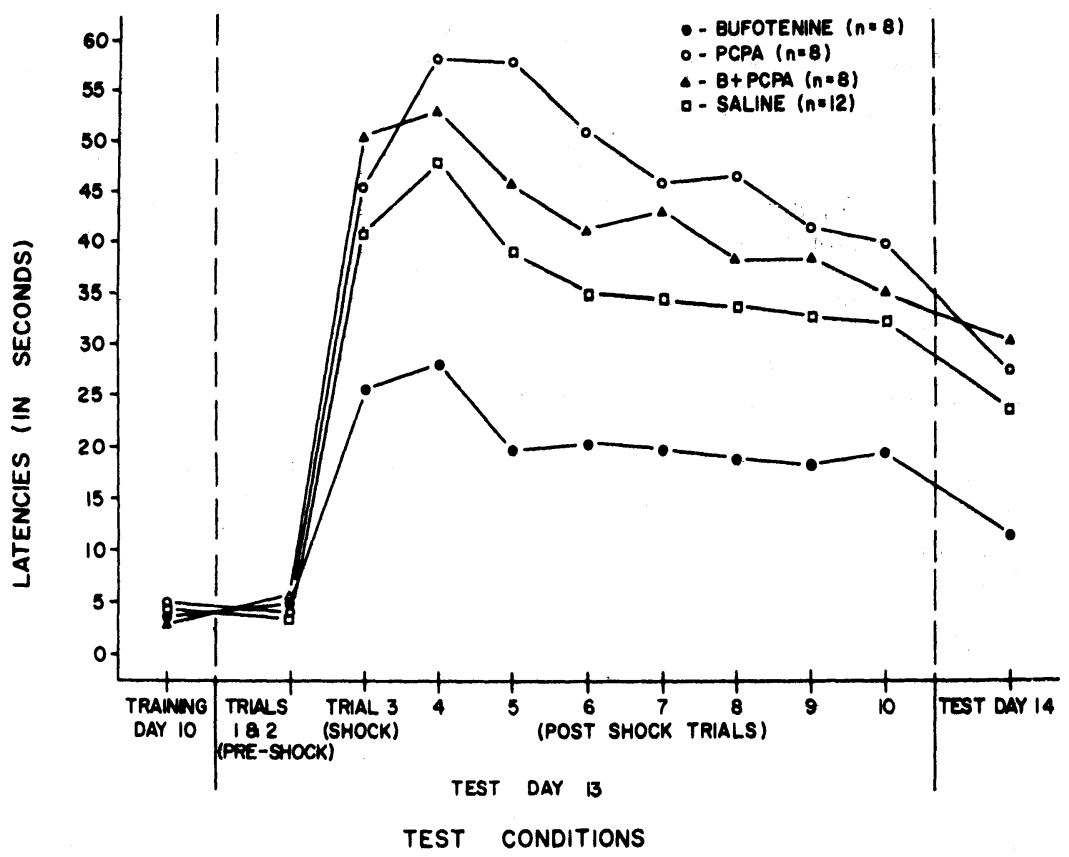

Fig. 1. Response latencies of all groups in Experiment I under all test conditions.

of photocells attached to BRS 200 series logic and a Hunter digital timer (Model 1522) were used to trigger the shock and provide accurate running times for each trial.

Procedure. At the beginning of the experiment, all Ss were placed on a $231 / 2-h$ water-deprivation schedule and handled for at least $10 \mathrm{~min}$ per day. A 5-day habituation period followed, in which Ss were allowed $30 \mathrm{~min}$ per day to explore the apparatus and drink water from a spout extending from a bottle attached to the back of the goalbox. Beginning on Day 6, 10 daily training trials were administered in which each animal was individually placed in the startbox and allowed to run down the alley to the goalbox. After about $10 \mathrm{sec}$ of drinking, the animal was returned to its cage for about $60 \mathrm{sec}$ to await the next trial. Training was administered at the same time for 5 consecutive days (i.e., Days 6-10).

Approximately $2 \mathrm{~h}$ after the last training trial, each $\mathrm{S}$ in Groups PCPA and B + PCPA received a single injection of PCPA at a dosage of $316 \mathrm{mg} / \mathrm{kg}$ and a volume of $.3 \mathrm{cc} / 100 \mathrm{~g}$ ). Saline Control Groups $a$ and $b$ received equal volumes of saline. No testing was conducted on Day 11, and Ss were watered for $30 \mathrm{~min}$ in their home cages. This procedure was followed to allow a 48-h period for the serotonin depletion effect of PCPA to attain maximal levels.

Thirty minutes before the beginning of testing on Day 12 , the B and B + PCPA groups were injected with $2.5 \mathrm{mg} / \mathrm{kg}$ bufotenine at a volume of $0.2 \mathrm{cc} / 100 \mathrm{~g}$ of bufotenine, while Saline Control Groups $b$ and $c$ received comparable volumes of saline. All Ss were then run in the apparatus in the usual manner. The purpose of this procedure was to ensure stable running rates under the drug conditions and to determine if any of the drugs per se affected running speed. Two hours following testing on Day 12, Groups PCPA and B + PCPA once again received single injections of PCPA and Saline Control Groups a and $\mathrm{b}$ received appropriate injections of saline. The second injection of PCPA was intended to maintain established low levels of serotonin.

On Day 13, Groups B and B + PCPA were injected with bufotenine and Saline Control Groups $b$ and $c$ received saline injections. Thirty minutes later, all Ss received two trials in the apparatus, conducted in the usual manner. For Trial 3, the equipment was programmed such that $\mathrm{S}$ received a $.5-\mathrm{mA}$ shock upon reaching the grid. The shock was terminated as soon as $S$ left the grid, and no further shock was introduced. Ss received seven more trials on Day 13.
On Day 14, Ss in Groups B and B + PCPA and Saline Control Groups $b$ and $c$ were appropriately injected, and $30 \mathrm{~min}$ later all animals received 10 trials in the usual manner.

\section{Results}

Mean running latencies for all groups under the various conditions are provided in Fig. 1. Running speeds rapidly stabilized during initial training so that by Day 5 there were no significant differences between groups. Moreover, the introduction of drugs had no effect on basic runway performance as reflected in the comparable running speeds for all groups during the first two preshock trials on the test day. Thus, any subsequent differences could not be attributed to differential running abilities of the groups during training or following drug administration.

The introduction of shock on Test Trial 3 produced the expected latency increase in the saline control group, 1 and indeed all groups were significantly slowed down in relation to their previous running speeds (all ps $<.01$; $t$ test of related means). However, the distractability of the B group was less than that of any of the other groups. Between-group comparisons showed that the average latency on Trial 3 for the B group was significantly lower than that of each of the other groups (all ps $<.05 ; t$ test of independent means), but that no other significant differences occurred.

An interesting development occurred on Test Trial 4, where the average latency for the PCPA group increased significantly over the Trial 3 measure $(t=3.28, d f=7$, $\mathrm{p}<.02$ ). No significant changes were observed in any of the other groups between Trials 3 and 4. A Duncan's multiple range test applied to the scores of Trials 4-10 revealed consistently significant differences between the PCPA and B groups (ps $<.05)$ and significant differences 
between the PCPA and saline groups on Trials 4-8, inclusive. Latencies for the PCPA group were generally higher than those of the B + PCPA group, but the differences were statistically significant only on Trials 5 and 6 (ps <.05). At no time were differences between the saline and B + PCPA groups significant ( $\mathrm{ps}>.05$ ).

It is clear from Fig. 1 that, while all groups showed some recovery during the seven postshock trials, latencies did not approach preshock levels. Furthermore, running speeds had not fully recovered by the end of testing on the following day, as can be seen from Fig. 1 . It is, however, noteworthy that the B group continued to run significantly faster than the other groups (all ps $<.05$ ) on the day following shock.

\section{EXPERIMENT II}

The results of Experiment I clearly indicated that bufotenine reduced the degree to which rats are distracted from ongoing behavior by an aversive stimulus. Conversely, distractability was very high in the PCPA group, whose latencies on the trials following footshock were significantly higher than those of the other three groups. One hypothesis which might explain these effects and which can be supported to some degree by the existing literature is that bufotenine decreases reactivity to electric shock (Evarts, Landau, Freygang, \& Marshall, 1955), while PCPA has the opposite effect (Tenen, 1967). Therefore, Experiment II was conducted to compare directly the effects of the two drugs on threshold levels and reactivity to footshock.

\section{Method}

Animals. Thirty-three naive male albino rats (Sprague-Dawley, Holtzman strain), weighing between 300 and $350 \mathrm{~g}$, were used as Ss. All animals were individually housed in wire mesh cages with food and water available at all times.

Apparatus. The test apparatus consisted of a $12 \times 12 \times 10$ in. box with a grid floor and clear Plexiglas walls. The grids consisted of 3/32-in. stainless steel rods spaced $1 / 2$ in. apart. Electric shock was delivered through the grid floor by a Lehigh Valley constant current shocker and scrambler (Model 113-04). Duration of shock and intershock intervals was controlled automatically by a Hunter electronic timer (Model 1512). Testing was conducted in a room with normal overhead illumination. External sounds were masked by the constant noise of the scrambler.

Procedure. The animals were divided randomly into $B(N=9)$ PCPA $(\mathrm{N}=8), \mathrm{B}+$ PCPA $(\mathrm{N}=8)$, and saline control $(\mathrm{N}=8)$ groups. Forty-eight hours after the test session, all Ss in the PCPA groups received intraperitoneal injections of PCPA $(316 \mathrm{mg} / \mathrm{kg})$, prepared in the manner described in Experiment $\mathrm{I}$. At the same time, the animals in the other groups received an equal volume of saline. Thirty minutes prior to testing, the B groups received a single injection of bufotenine $(2.5 \mathrm{mg} / \mathrm{kg})$, while the other groups received equal-volume injections of saline. In this way, number of injections and total volume was held constant between groups.

Each $S$ received one test session, beginning with a 2-min familiarization period in the test apparatus. The animal then received an ascending series of 24 electric shocks ranging between .01 and $.80 \mathrm{~mA}$. For the first 10 shocks, current levels were increased by $.01-\mathrm{mA}$ steps and by $.05-\mathrm{mA}$ steps thereafter. Following a 2-min rest interval, the same series of shocks was
Table 1

Median Response Thresholds for All Groups in Experiment II

\begin{tabular}{lcccc}
\hline & $\begin{array}{c}\text { Bufo- } \\
\text { tenine }\end{array}$ & PCPA & $\begin{array}{c}\text { B + } \\
\text { PCPA }\end{array}$ & Saline \\
\hline Flinching & .20 & .08 & .09 & .08 \\
Squealing & .33 & .22 & .37 & .26 \\
Moving & .78 & .33 & .41 & .20 \\
\hline
\end{tabular}

administered in descending order. Each shock was $1 \mathrm{sec}$ in duration, with an intershock interval of $30 \mathrm{sec}$.

A procedure was employed in which the $\mathrm{E}$ observing the animals' behavior was not aware of the treatment conditions. Responses were scored according to the following categories: flinching (startle response in which the rear paws do not leave the grid floor), squealing, moving (lomotion involving all four legs), and jumping (both rear paws leave the floor). These categories were not intended to be mutually exclusive, and frequently several responses were recorded following one shock. An insufficient number of jumping responses were recorded to enable meaningful statistical comparisons, and, as a result, no data are provided for this category. Defecation and urination data were also recorded to provide indices of fear.

Ascending and descending thresholds were determined for each category. In the ascending series, a response threshold was calculated as the lowest shock level associated with the first three consecutive responses. A descending threshold was calculated as the shock level preceding three consecutive shocks which produced no response in that particular category. In a few instances, animals failed to meet one or the other criterion, and they were assigned arbitrary threshold values of .85 in the ascending series or .00 in the descending series. The average of the ascending and descending values was defined as the S's threshold for a response category. The data and statistical comparisons to be reported are based on the average threshold scores.

\section{Results}

As can be seen from Table 1, response thresholds for the B group were generally higher than those of the other three groups. A Kruskal-Wallis one-way analysis of variance applied to scores within the three categories revealed significant between-group differences for the flinching $(\mathrm{H}=13.13, \mathrm{p}<.01)$ and moving $(\mathrm{H}=8.76$, $\mathrm{p}<.05)$ responses. In both cases, the differences were due to the high thresholds of the $B$ groups relative to those of the other groups (all ps $<.05$; Mann-Whitney $\mathrm{U}$ test). There was also a significant difference in moving threshold between the B + PCPA and saline groups ( $=$ $5, p<.002$ ), but no other significant differences occurred in response thresholds.

In view of the recent suggestion that PCPA increases reactivity to shock at above-threshold levels without directly affecting threshold values (Fibiger, Mertz, \& Campbell, 1972), an analysis was made of the reactions of the PCPA and saline groups as a function of shock level. The ascending and descending shock series were collapsed into eight blocks of three shock levels, thereby providing for a maximum of six responses per animal per block. The responses of the two groups are plotted in Fig. 2, where the arrow along the abscissa denotes the shock range within which threshold values were obtained for both groups. A Kilomogrov-Smirnov two-sample test 


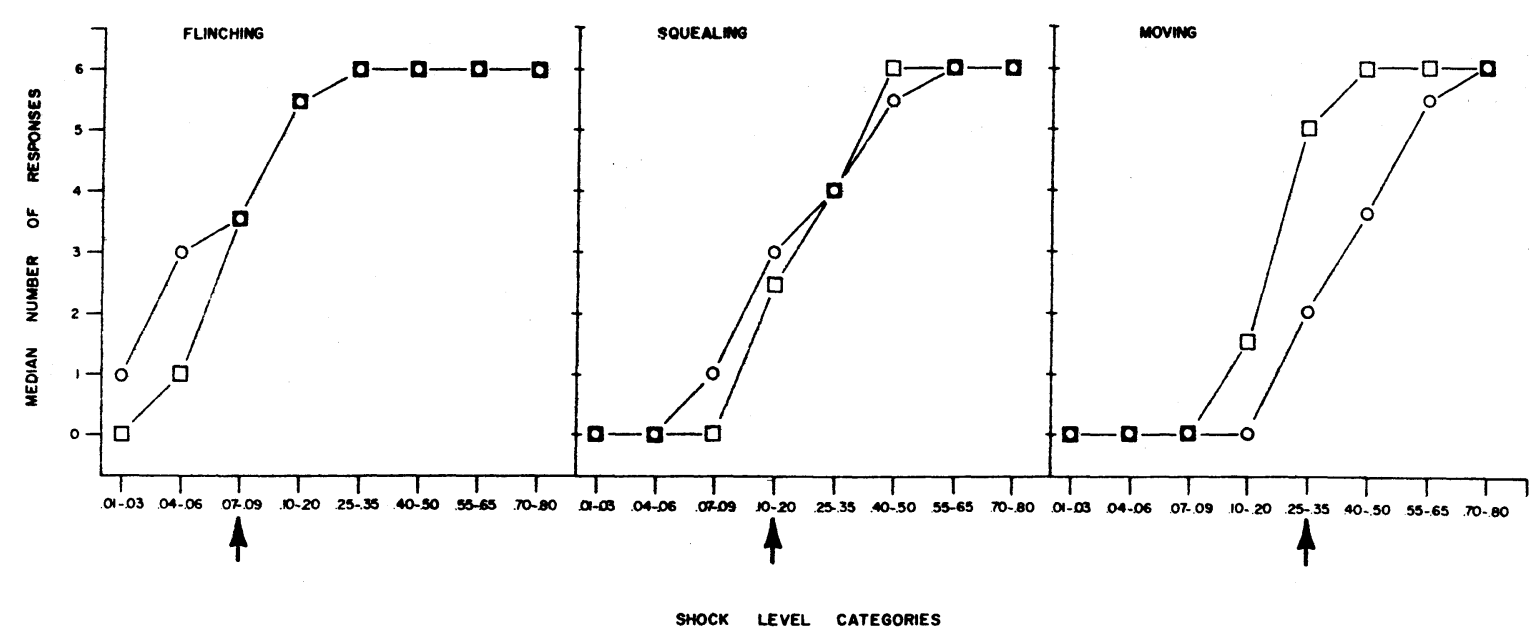

Fig. 2. Responses of PCPA and saline groups as a function of shock intensities in Experiment II. Arrows denote threshold ranges.

was used to analyze the data in the below-threshold categories as well as the values in the above-threshold categories. The only significant difference occurred in the moving response measure, but, contrary to the predictions of Fibiger et al (1972), the PCPA group moved less than the controls in response to above-threshold shocks $\left(\mathrm{K}_{\mathrm{D}}=6, \mathrm{p}<.01\right.$; two-tailed). Thus, in the present conditions, PCPA did not appear to alter threshold values, and, at above-threshold intensities, the PCPA group, if anything, was less active following shock.

Table 2 provides elimination measures which may be taken as general indices of emotionality. There were no significant differences in shock values at which defecation first occurred, but overall boli counts were lower in the B group than in the saline group $(U=11$, $\mathrm{p}<.02$ ). It was not possible to obtain reliable measures of urination frequency, but, as can be seen from Table 2, shock values at which urination first occurred were higher in the $B$ group than in the saline $(U=15.5$, $\mathrm{p}<.05$; one-tailed $)$ and PCPA $(\mathrm{U}=1, \mathrm{p}<.002)$ groups.

\section{DISCUSSION}

The present results, which demonstrated lowered reactivity to footshock in the $B$ groups clearly reflect the desensitization properties of bufotenine. The results add further support to the general hypothesis that bufotenine interferes with sensory or perceptual functioning, although alternative interpretations can be considered. For example, the relatively fast running speeds of the B group following shock in Experiment I could reflect heightened activity due to a direct effect on motivational or motor mechanisms. However, an interpretation in terms of exaggerated drive states is improbable on the basis of previous research in which $B$ groups were no different from controls in learning goal-directed responses under similar hunger conditions
(Winocur et al, 1971). It is also unlikely that bufotenine caused an increase in general locomotion or a loss of response inhibition, since in previous investigations Es have emphasized the attenuating effects of bufotenine on locomotor behavior (Evarts, 1956; Winocur et al, 1969).

In a recently completed study ${ }^{2}$ examining orienting behavior, further evidence was obtained in support of a bufotenine-induced attentional dysfunction. The results showed that bufotenine increased the latencies of orienting responses as well as reducing their strength and frequency of occurrence. There was no evidence that the B. groups were physically incapable of performing the orienting response; rather, it appeared that the animals were not efficiently receiving or processing the sensory input. In line with this hypothesis are electrophysiological studies which have shown that bufotenine restricts synaptic transmission along major sensory systems as well as higher levels of the central nervous system (Evarts et al, 1955; Marazzi \& Hart, 1955).

A different picture emerges with respect to PCPA. In Experiment I, from Trial 4 onwards, the PCPA group displayed consistently slower latencies than the other groups. This finding might be expected on the basis of published reports linking PCPA with decreased activity (Tenen, 1967) and heightened reactivity to footshock

Table 2

Median Shock Levels at Which Defecation and Urination First Occurred in Experiment II

\begin{tabular}{ccccc}
\hline & $\begin{array}{c}\text { Bufo- } \\
\text { tenine }\end{array}$ & PCPA & PCPA & Saline \\
\hline Defecation & .40 & .53 & .53 & .45 \\
& $(3)$ & $(5)$ & $(5)$ & $(5.6)$ \\
Urination & .75 & .40 & .65 & .50 \\
\hline
\end{tabular}

Note-Numbers in parentheses refer to median number of boli produced by the various groups. 
(Fibiger et al, 1972). The results of Experiment II appear to discount an interpretation in terms of decreased sensitivity to painful stimuli, but they do provide some support for an attenuated-activity hypothesis. However, a difficulty with the latter interpretation is the fact that the PCPA group in Experiment I displayed normal preshock running behavior. Furthermore, there are a number of reports in the literature which cannot be explained in terms of reduced activity. For example, several investigators have shown that PCPA facilitates various types of learning, including active avoidance conditioning (Tenen, 1967; Brody, 1970) and discrimination learning (Stevens, 1970).

An alternative explanation, favored by Brody (1970), which may account for the apparently beneficial effects of PCPA as well as the present results is that PCPA renders an animal more responsive to external stimuli, thereby facilitating the selection of highly adaptive behavioral patterns. During the preshock trials of Experiment I, rapid goal-oriented behavior was clearly the most appropriate, while, following the shock, cautious slower running following the experience of an unexpected painful stimulus could be regarded as highly adaptive. In Experiment II, response thresholds were not affected by PCPA, but the PCPA group displayed less movement at higher shock intensities, perhaps reflecting once again an adaptive strategy of response suppression. This type of hypothesis could also account for the superior performance of PCPA-injected animals on tasks which require incompatible forms of behavior, e.g., active and passive avoidance conditioning (Brody, 1970).

The failure to observe lowered response thresholds in the PCPA group of Experiment II is consistent with the results of Fibiger et al (1972), although these authors found greater rather than less activity at higher shock levels. However, there were important procedural differences between the Fibiger et al and the present experiments. In the Fibiger et al study, animals could always escape (but not avoid) shock by running over to a second compartment. In these circumstances, it can be argued that the PCPA group responded adaptively at higher shock levels by escaping more rapidly than controls. In our situation, inescapable shock was delivered in a single, closed compartment, which could be expected to produce different response patterns.

Thus, it can be argued that while bufotenine reduces the animal's ability to respond effectively to environmental events, the opposite effect seems to be produced by PCPA. Further support for this position is provided by the B + PCPA groups, which in all conditions of the present research behaved like saline control groups. In Experiment $I$, the characteristic effects of bufotenine and PCPA were effectively cancelled out in the B + PCPA group, and, in Experiment II, higher threshold values associated with bufotenine were lowered to normal levels by the addition of PCPA. Similar patterns were observed for the elimination data of Experiment II.
The mutually counteracting effect between bufotenine and PCPA confirms the earlier report by Winocur et al (1971) and appears similar to the relationship between PCPA and 5-hydroxytryptophan (5HTP), the serotonin precursor. Tenen (1967) and Hartmann and Geller (1971) demonstrated that 5HTP reversed the effects of PCPA on a number of measures, including avoidance conditioning, pain sensitivity, and conditioned fear behavior. Tenen did not observe this reversal using serotonin (5hydroxytryptamine), which does not pass through the blood-brain barrier, and attributed PCPA-induced behavioral changes to a central rather than peripheral nervous system effect. It has been established that bufotenine crosses the blood-brain barrier (Curtis, 1966), so that it would appear that the present results similarly reflect disruption of normal central nervous system activity. This conclusion is in line with our previous suggestion (Winocur et al, 1971) that bufotenine alters behavior by disrupting some phase of normal metabolism of brain serotonin.

\section{REFERENCES}

Brody, J. F. Behavioral effects of serotonin depletion and of p-chlorophenylalanine (a serotonin depletor) in rats. Psychopharm acologia, 1970, 17, 14-33.

Curtis, D. R. The effect of some psychomimetic drugs upon single nerve cells. In M. Rinkel (Ed.), Biological treatment of mental illness. New York: Page, 1966. Pp. 243-261.

Evarts, E. C. Some effects of bufotenine and LSD on the monkey. A.M.A. Archives of Neurology \& Psychiatry, 1956, 75, 14-33.

Evarts, E. C., Landau, W., Freygang, W., \& Marshall, W. H. Some effects of LSD and bufotenine on electrical activity of the cat's visual system. American Journal of Physiology, 1955, $182,594-598$.

Fabing, H. D., \& Hawkins, J. R. Intravenous bufotenine injection in the human being. Science, 1966, 123, 886-887.

Fibiger, H. C. Mertz, P. H., \& Campbell, B. A. The effect of para-chlorophenylalanine on aversion thresholds and reactivity to foot shock. Physiology \& Behavior, 1972, 8, 250-263.

Hartmann, R. J., \& Geller, I. P-chlorophenylalanine effects on a conditioned emotional response in rats. Life Sciences, 1971, $10,927-933$.

Himwich, H. E., \& Brune, G. G. Relationship between indemetabolism and schizophrenic behavior. In $M$. Rinkel (Ed.), Biological treatment of mental illness. New York: Page, 1966. Pp. 284-302.

Koe, B. K., \& Weissman, A. p-Chlorophenylalanine: A specific depletor of brain serotonin. Journal of Pharmacology \& Experimental Therapeutics, 1966, 154, 499-516.

Maire, F. W., \& Hixon, A. C. Behavioral and performance changes in trained rats following bufotenine injection. International Journal of Neuropsychiatry, 1966, 2, 135-142.

Marazzi, A. S., \& Hart, E. R. Relationship of hallucinogens to adrenergic cerebral synapses. Science, 1955, 121, 265.

Stevens, D. A. The effects of p-chlorophenylalanine on behavior: III. Facilitation of brightness discrimination in satiated rats. Life Sciences, 1970, 9, 1127-1134.

Tenen, S. S. The effects of p-chlorophenylalanine, a serotonin depletor, on avoidance acquisition, pain sensitivity, and related behavior in the rat. Psychopharmacologia, 1967, 10, 204-219.

Winocur, G., Bagchi, S. P., \& Hubbard, P. Effects of bufotenine and p-chlorophenylalanine on stress induced behavior. Psychopharmacologia, 1971, 22, 100-110.

Winocur, G., Bagchi, S. P., \& Y oung, J. D. Beahvioral changes in rats following bufotenine injection. Perceptual \& Motor Skills, $1969,28,527-533$.

\section{NOTES}

1. The various saline control groups did not differ significantly on any measure, and so their scores were combined to form a single saline group.

2. Miskiman, D. E., Winocur, G., and Bagchi, S. P. Disruption of orienting behaviour following bufotenine injection. In preparation.

(Received for publication June 8, 1973; revision received December 6,1973 .) 